\title{
Expression of SARS-CoV-2 host cell entry factors in immune system components of healthy individuals and its relevance for COVID-19 immunopathology
}

\author{
Ashutosh Kumar ${ }^{1}$, Ravi Narayan ${ }^{1}$, Sujeet Kumar², Chiman Kumari ${ }^{3}$, Vikas Pareek ${ }^{4}$, and \\ Pranav Prasoon ${ }^{5}$ \\ ${ }^{1}$ All India Institute of Medical Sciences - Patna \\ ${ }^{2}$ Amity Institute of Biotechnology, Amity University \\ ${ }^{3}$ Post Graduate Institute of Medical Education and Research \\ ${ }^{4}$ National Brain Research Centre \\ ${ }^{5}$ University of Pittsburgh School of Medicine
}

August 4, 2020

\begin{abstract}
Introduction: Intense immunological dysregulation including immune cell lesions have been characteristically observed in severe cases of Coronavirus Disease-2019 (COVID-19), for which molecular mechanisms are least understood. A study of physiological expressions of SARS-CoV-2 host cell entry related factors in immune system components may help explain molecular mechanisms involved in COVID-19 immunopathology. Materials and Methods: Transcriptomic and proteomic expression metadata for SARS-CoV-2 host cell entry receptor ACE2 and entry associated proteases (TMPRSS2, CTSL, and FURIN) were analysed in silico across immune system components including blood lineage cells. Results: ACE2 was not-detected in any of the studied immune cell components, however, varying transcriptomic and proteomic expressions were observed, for TMPRSS2, CTSL, and FURIN. Conclusions: Non-detectable expressions of SARS-CoV-2 host cell entry receptor ACE2 in immune system components or blood lineage cells indicate it doesn't mediate immune cell lesions in COVID-19. Alternative mechanisms need to be explored for COVID-19 immuno-pathogenesis.
\end{abstract}

\section{Keywords: COVID-19, SARS-Cov-2, ACE2, TMPRSS2, Coronavirus, Immunopathology}

\section{Introduction}

The ongoing pandemic of COVID-19 caused by the novel strain of coronavirus, $S$ evere $A$ cute $R$ espiratory $S$ yndrome Co ronavirus-2 (SARS-CoV-2) has affected millions of lives globally. SARS-CoV-2, an enveloped, positive sense, single stranded RNA virus belongs to genus betacoronavirus of the Coronaviridae family that includes, SARS-CoV-1 and MERS-CoV; the causative agents of respiratory syndrome outbreaks in recent past. ${ }^{1}$ Receptor recognition by viruses being the foremost step of viral infections is of paramount importance in determining the severity of infections. In the case of SARS-CoV-2, the viral entry to host cells is reported to be primarily mediated by receptor binding domain (RBD) of the viral $\mathrm{S}$ (spike) protein to a cell surface receptor ACE2. The recpetor recognition and the ensuing fusion are critical for viral infections that is facilitated by the entry associated protein transmembrane serine protease 2 (TMPRSS2) or Cathepsin L (CTSL) in concert with FURIN, a host-cell associated protease that mediates the cleavage of the viral spike (S) protein. ${ }^{2,3}$

Epithelilal cells enriched in ACE2 expression are slectively biased for bearing the viral load and the COVID-19 infections are primarily attracted to lungs via the nasal route, primarily transmitted among people through 
respiratory droplets and contact routes. The studies indicated organ specific pathogenesis by the SARSCoV-2 binding-mediated dysregulation of ACE2 (ref). Human ACE2 is an interferon stimulated gene, and thus SARS-CoV-2 may also exploit cell type-specific interferon-driven upregulation of ACE2. ${ }^{4}$

In severe cases of COVID-19 pathogenesis, a hyperactive innate-immune response characterised by very high level of proinflammation markersknown as 'cytokine storm' is observed in lungs as well as other organs. The COVID-19 severity is also characterised by differential response of immune cells; reflected by increased counts of neutrophils and macrophages, however, reduced counts of eosinophil and lymphocytes (specifically of CD4+ and CD8+ T cells). ${ }^{5,6}$ An increased death of CD4+ and CD8+ T lymphocytes is also a very frequent finding in severe COVID-19. ${ }^{5,7}$

To delineate, how the immune system components; especially immune related blood-cells, are affected by SARS-CoV-2 infections of an individual, is expected to provide insights on molecular mechanisms involved in immunological dysregulations in COVID-19. Whether the immune system components are potential targets of SARS-CoV-2 or not, would depend on the immune system (cell-types) expression of the receptor ACE2, and the associated cell-entry factors (TMPRSS2 and FURIN). Although, many sporadic reports are available, ${ }^{4,8,9}$ to our knowledge, this has not been addressed comprehensively, so far. By utilizing the standard databases for transcriptomics (tissue and single cell) and proteomic expressions in human immunesystem components; we have performed in silico analysis for evaluating the roles of the expression-levels of host cell-entry factors (ACE2, TMPRSS2, CTSL, and FURIN) for the facilitated entry of SARS-CoV-2 that would lead to immune dysfunctions.

\section{Materials and Methods}

We performed in silico analysis of mRNA and protein expressions of ACE2, TMPRSS2, CTSL, and FURIN in human immune system components (bone marrow, bone marrow, and lymphoid tissue - thymus, appendix, spleen, lymph nodes, spleen, tonsil) using tissue transcriptome and immunohistochemistry (IHC) data available in Human Protein Atlas (HPA) (https://www.proteinatlas.org/). Further, mRNA expression across blood cell types were analyzed using single cell RNA sequencing data available in 'The Blood Atlas (a section of HPA)'.

\section{External data source methods}

Estimation of mRNA expression and localization of human proteins were performed by the source laboratory using tissue and single cell deep sequencing of RNA (RNA-seq) and IHC in normal tissue.

\section{IHC}

As described by the source labs, specimens containing normal tissue were collected and sampled from anonymized paraffin embedded material of surgical specimens, in accordance with approval from the local ethics committee. The specimens were derived from surgically removed tissue, normal was defined by tissue specific morphological parameters and absence of neoplasia. IHC staining was performed on normal tissue microarray using a standard protocol (https://www.proteinatlas.org/download/IHC_protocol.pdf). Antibodies against human ACE2 (HPA000288, CAB026174), TMPRSS2 (HPA035787), CTSL (CAB000459), and FURIN (CAB009499) were labeled with DAB (3, 3'-diaminobenzidine) stain. Protein expression score was calculated for each receptor based on the staining intensity (negative, weak, moderate or strong) and fraction of stained cells $(<25 \%, 25-75 \%$ or $>75 \%)$. For each protein, the IHC staining profile was matched with mRNA expression data and gene/protein characterization data to yield an 'annotated protein expression' profile.

\section{Tissue transcriptomics}

HPA collects transcriptomic data from the three databases (HPA, GTEx and FANTOM5). For HPA RNAseq total RNA was extracted from the tissue samples healthy individuals (Accession no: PRJEB4337, Ensembl: ENSG00000130234 (version 92.38) using the RNeasy Mini Kit (Qiagen, Hilden, Germany) according to the manufacturer's instructions. The extracted RNA samples were analyzed using either an Experion automated 
electrophoresis system (Bio-Rad Laboratories, Hercules, CA, USA) with the standard-sensitivity RNA chip or an Agilent 2100 Bioanalyzer system (Agilent Biotechnologies, Palo Alto, USA) with the RNA 6000 Nano Labchip Kit. Sample samples of high RNA quality (RNA Integrity Number > 7.5) were used for the mRNA sequencing. mRNA sequencing was performed on Illumina HiSeq2000 and 2500 machines (Illumina, San Diego, CA, USA) using the standard Illumina RNA-seq protocol (with a read length of 2x100 bases). For estimation of transcript abundance Kallisto v0.43.1 was used (https://pachterlab.github.io/kallisto/about). The normalized Tags Per Million (TPM) for each gene from the three databases were calculated and included in the Human Protein Atlas. Each tissue was categorized for the intensity of gene expression using a cutoff value of $1 \mathrm{NX}$ as a limit for detection across all tissues. A tissue was categorized (i) enriched if it had NX level at least four times higher than other tissues, (ii) low specificity if NX [?] 1 in at least one tissue, (iii) Not detected if $\mathrm{NX}<1$ in all tissues.

\section{Single cell transcriptomics}

Single cell transcriptomic data covering various $\mathrm{B}$ and $\mathrm{T}$ cells, monocytes, granulocytes and dendritic cells were analyzed. "The Blood Atlas' collects data from the three datasets (HPA (https://www.proteinatlas.org/humanproteome/blood), Monaco et al ${ }^{10}$, and Schmiedel et al ${ }^{11}$ ). HPA presents data for 18 cell types present in human blood that were isolated with cell sorting followed by RNA-seq analysis. Annotation of the studied genes was done according to cell type specificity (cell type enriched, group enriched, cell type enhanced and low cell type specificity) and cell type distribution (detected in single, few, many or all cell types).

Further details of the assays and annotation used by the Human Protein Atlas can be accessed at:https://www.proteinatlas.org/about/assays+annotation\#ihk.

\section{Meta-data analysis}

We analysed HPA transcriptomic and proteomic data in respect of tissue/cell type specific expression in immune system components. Graphs were plotted and inferences were made based on the final results.

\section{Results \& Discussion}

We have performed in silico analysis of meta-data for transcriptomic and proteomic expression of SARSCoV-2 host cell entry related factors (ACE2, TMPRSS2, CTSL, and FURIN). SARS-CoV-2 host cell entry receptor ACE2 was not-detected in bulk-transcriptomic and proteomic expression analysis in any of the studied immune cell components (bone marrow and lymphoid tissue) (Table 1, Fig. 1). ACE2 was also not-detected in single cell transcriptomic analysis of blood cell lineages (Table 2, Fig. 2). 'Not-detected' to 'enriched' transcriptomic expression, were observed in bulk (bone marrow and lymphoid tissue) as well as in single cell analysis (blood cell lineages), and 'not-detected' to 'medium level' proteomic expression (bone marrow and lymphoid tissue) expression were observed, for SARS-CoV-2 host cell entry associated proteases TMPRSS2, CTSL, and FURIN (Table 1-2, Fig. 1-2).

The observations made in this study are important and provide insights regarding immunopathogenesis in COVID-19. We findings, the non-detectable level of expressions of viral host cell entry receptor ACE2, and non-detectable to medium range expression of host cell entry associated proteases TMPRSS2, CTSL, and FURIN across immune system components of healthy individuals (Table 1-2, Fig. 1-2) are in line with recent sporadic studies reporting expression of a single or a set of these factors across human tissues. ${ }^{4,8,9}$ The nondetectable expressions of ACE2 across immune system components including blood (immune) cells indicate their non-susceptibility for SARS-CoV-2 infection. However, recent studies have provided indications of immune cell lesion in COVID-19. ${ }^{12-15}$ Lymphocytopenia in COVID-19 cases is marked by selective killing of T cells. ${ }^{12}$ Moreover, SARS-CoV-2 mediated atrophy and lesion of the human lymphoid tissue - like spleen and lymph nodes-, also have been observed in a recent study. ${ }^{16}$ The viral mediated manipulations of the host immune-cell receptor signaling is well established fact; however, any such evidence specific to SARS$\mathrm{CoV}-2$ is currently scarce in literature. ${ }^{17}$ In absence of significant cell-surface expression of ACE2 in immune system components, the alternative mechanisms which are exploited by the COVID-19 virus to induce 
immunopathology, need to be ascertained. Therefore, our finding warrants for a systematic review of existing literature indicated for the set of plausible mechanisms which may explain immunological dysregulation in COVID-19 without the involvement of ACE2 signaling. The available data suggets that either, (i) selective killing of $\mathrm{T}$ cells may be a resultant of high productions of cytokines and pro-inflammatory markers, ${ }^{15,18}$ (ii) direct viral protein toxicity, ${ }^{16}$ (iii) or presence of an additional SARS-CoV-2 cell entry receptor which is expressed in immune system components. ${ }^{19}$ Recently, a study proposed Neuropilin-1 (NRP1) as an alternative receptor for SARS-CoV-2 cell entry, which has significant expression in immune cells. ${ }^{19,20}$ However, further in situ/in vivo studies will be necessary to prove that NRP1 can mediate SARS-CoV-2 infection of immune cells.

\section{Acknowledgements}

Authors express gratitude to 'The Human Protein Atlas' for allowing use of its metadata for this study.

\section{Competing Interests}

The authors declare no competing interests.

\section{Funding Declaration}

No substantial funding was obtained for this work.

\section{Author (s) Contributions}

AK conceived the idea and wrote the first draft. RKN analyzed the data and prepared figures and tables. SK, RKN, CK, and PP reviewed and edited for the final draft.

\section{References}

1 Jaimes JA, André NM, Chappie JS, et al. Phylogenetic Analysis and Structural Modeling of SARS-CoV-2 Spike Protein Reveals an Evolutionary Distinct and Proteolytically Sensitive Activation Loop.J Mol Biol 2020;432:3309-25. doi:10.1016/j.jmb.2020.04.009

2 Hoffmann M, Kleine-Weber H, Schroeder S, et al. SARS-CoV-2 Cell Entry Depends on ACE2 and TMPRSS2 and Is Blocked by a Clinically Proven Protease Inhibitor. Cell 2020;181 :271-280.e8. doi:10.1016/j.cell.2020.02.052

3 Hoffmann M, Kleine-Weber H, Pöhlmann S. A Multibasic Cleavage Site in the Spike Protein of SARS-CoV-2 Is Essential for Infection of Human Lung Cells. Mol Cell 2020;78 :779-784.e5. doi:10.1016/j.molcel.2020.04.022

4 Ziegler CGK, Allon SJ, Nyquist SK, et al. SARS-CoV-2 receptor ACE2 is an interferon-stimulated gene in human airway epithelial cells and is detected in specific cell subsets across tissues. CellPublished Online First: 27 April 2020. doi:10.1016/j.cell.2020.04.035

5 Kumar A, Prasoon P, Shekhawat PS, et al. Pathogenesis Guided Therapeutic Management of COVID-19: An Immunological Perspective.SSRN Electron J Published Online First: 15 June 2020. doi:10.2139/ssrn.3626907

6 Pedersen SF, Ho YC. SARS-CoV-2: A storm is raging. J. Clin. Invest. 2020;130 :2202-5. doi:10.1172/JCI137647

7 Tay MZ, Poh CM, Rénia L, et al. The trinity of COVID-19: immunity, inflammation and intervention. Nat. Rev. Immunol. 2020;:1-12. doi:10.1038/s41577-020-0311-8

8 Sungnak W, Huang N, Bécavin C, et al. SARS-CoV-2 entry factors are highly expressed in nasal epithelial cells together with innate immune genes. Nat Med 2020;:1-7. doi:10.1038/s41591-020-0868-6

9 Muus C, Luecken MD, Eraslan G, et al. Integrated analyses of single-cell atlases reveal age, gender, and smoking status associations with cell type-specific expression of mediators of SARS-CoV-2 vi- 
ral entry and highlights inflammatory programs in putative target cells.bioRxiv 2020;:2020.04.19.049254. doi:10.1101/2020.04.19.049254

10 Monaco G, Lee B, Xu W, et al. RNA-Seq Signatures Normalized by mRNA Abundance Allow Absolute Deconvolution of Human Immune Cell Types. Cell Rep 2019;26 :1627-1640.e7. doi:10.1016/j.celrep.2019.01.041

11 Schmiedel BJ, Singh D, Madrigal A, et al. Impact of Genetic Polymorphisms on Human Immune Cell Gene Expression. Cell2018;175 :1701-1715.e16. doi:10.1016/j.cell.2018.10.022

12 Zhang X, Tan Y, Ling Y, et al. Viral and host factors related to the clinical outcome of COVID-19. Nature 2020;:1-7. doi:10.1038/s41586-020-2355-0

13 Tan L, Wang Q, Zhang D, et al. Lymphopenia predicts disease severity of COVID-19: a descriptive and predictive study. Signal Transduct. Target. Ther. 2020;5 :1-3. doi:10.1038/s41392-020-0148-4

14 Lippi G, Henry BM. Eosinophil count in severe coronavirus disease 2019. QJM . 2020;113(7):511-512. doi:10.1093/qjmed/hcaa137

15 Diao B, Wang C, Tan Y, et al. Reduction and Functional Exhaustion of T Cells in Patients With Coronavirus Disease 2019 (COVID-19). Front Immunol 2020;11 :827. doi:10.3389/fimmu.2020.00827

16 Feng Z, Diao B, Wang R, et al. The Novel Severe Acute Respiratory Syndrome Coronavirus 2 (SARS-CoV-2) Directly Decimates Human Spleens and Lymph Nodes. medRxiv2020;2 :2020.03.27.20045427. doi:10.1101/2020.03.27.20045427

17 Ong EZ, Chan KR, Ooi EE. Viral Manipulation of Host Inhibitory Receptor Signaling for Immune Evasion. PLoS Pathog. 2016;12 . doi:10.1371/journal.ppat.1005776

18 Channappanavar R, Perlman S. Pathogenic human coronavirus infections: causes and consequences of cytokine storm and immunopathology. Semin. Immunopathol. 2017;39 :529-39. doi:10.1007/s00281-017-0629$\mathrm{x}$

19 Daly JL, Simonetti B, Antón-Plágaro C, et al. Neuropilin-1 is a host factor for SARS-CoV-2 infection. bioRxiv2020;:2020.06.05.134114. doi:10.1101/2020.06.05.134114

20 NRP1 protein expression summary - The Human Protein Atlas. https://www.proteinatlas.org/ENSG00000099250-NRP1 (accessed 13 Jul 2020).

\section{Figures and Tables}
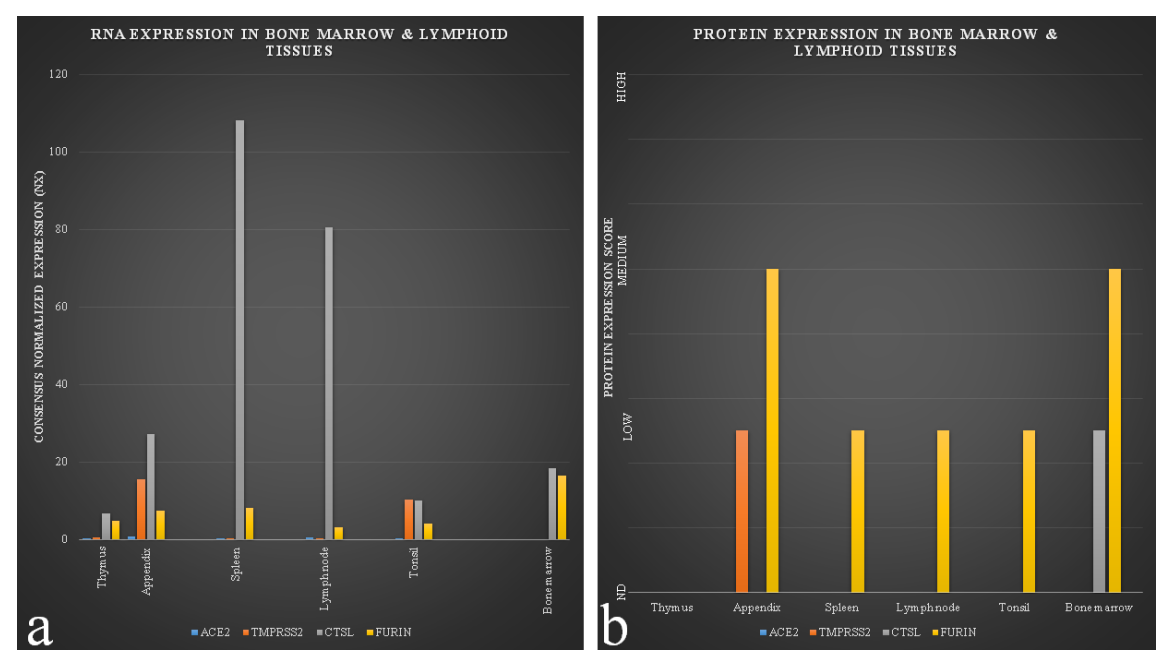
Figure 1 RNA and Protein expression of SARS-CoV-2 cell entry related factors in bone marrow and lymphoid tissues.

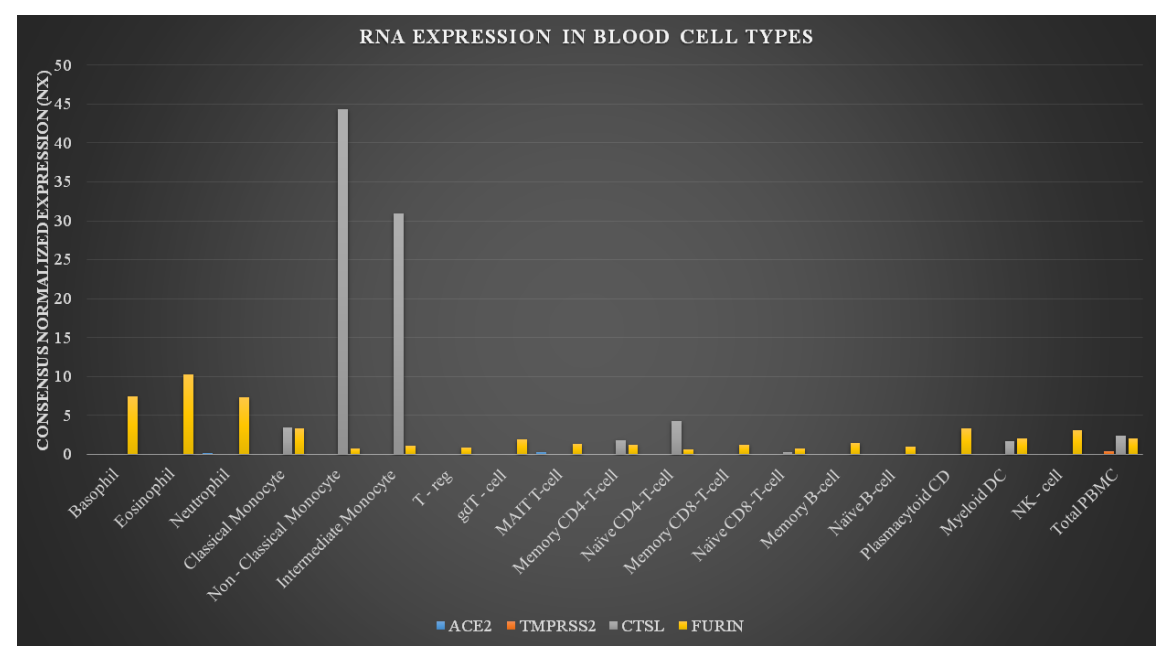

Figure 2 RNA expression (NX) ${ }^{*}$ of SARS-CoV-2 cell entry related factors in blood cell types.

Table 1 RNA and Protein expression of SARS-CoV-2 cell entry related factors in bone marrow and lymphoid tissues.

\begin{tabular}{lllllll}
\hline Tissue & & Thymus & Appendix & Appendix & Spleen & S1 \\
\hline Cellular components & & & Glandular cells & Lymphoid Tissue & Cells in red pulp & C \\
RNA Expression (NX) & ACE2 & 0.2 & 0.8 & 0.8 & 0.2 & 0. \\
& TMPRSS2 & 0.5 & 15.5 & 15.5 & 0.3 & 0. \\
& CTSL & 6.8 & 27.2 & 27.2 & 108.1 & 10. \\
Protein Expression & FURIN & 4.8 & 7.5 & 7.5 & ND & ND \\
& ACE2 & ND & ND & ND & ND \\
& TMPRSS2 & ND & Low & ND & ND \\
& CTSL & ND & ND & ND & Low & N \\
& FURIN & ND & Medium & ND & & \\
\end{tabular}

${ }^{*} \mathrm{NX}$ - normalized expression, ${ }^{* *} \mathrm{ND}$ - not detected

Table 2 RNA expression (NX)*of SARS-CoV-2 cell entry related factors in blood cell types.

\begin{tabular}{lllll}
\hline BLOOD CELLS & ACE2 & TMPRSS2 & CTSL & FURIN \\
\hline Basophil & 0 & 0 & 0 & 7.5 \\
Eosinophil & 0 & 0 & 0 & 10.3 \\
Neutrophil & 0.2 & 0 & 0 & 7.3 \\
Classical Monocyte & 0 & 0 & 3.5 & 3.3 \\
Non - Classical Monocyte & 0 & 0 & 44.3 & 0.7 \\
Intermediate Monocyte & 0 & 0 & 31 & 1.1 \\
T - reg & 0 & 0 & 0 & 0.9 \\
gdT - cell & 0 & 0 & 0 & 1.9 \\
MAIT T-cell & 0.3 & 0 & 0 & 1.4 \\
Memory CD4-T-cell & 0 & 0 & 1.8 & 1.2
\end{tabular}




\begin{tabular}{lllll}
\hline BLOOD CELLS & ACE2 & TMPRSS2 & CTSL & FURIN \\
\hline Naïve CD4-T-cell & 0 & 0 & 4.3 & 0.6 \\
Memory CD8-T-cell & 0 & 0 & 0 & 1.2 \\
Naïve CD8-T-cell & 0 & 0 & 0.3 & 0.8 \\
Memory B-cell & 0 & 0 & 0 & 1.5 \\
Naïve B-cell & 0 & 0 & 0 & 1 \\
Plasmacytoid CD & 0 & 0 & 0 & 3.3 \\
Myeloid DC & 0 & 0 & 1.7 & 2.1 \\
NK - cell & 0 & 0 & 0 & 3.1 \\
Total PBMC & 0 & 0.4 & 2.4 & 2 \\
\hline
\end{tabular}

${ }^{*} \mathrm{NX}$ - normalized expression 